\begin{tabular}{ccc}
\hline & International Journal of Engineering \& Technology, $7(2.12)(2018) 93-96$ \\
SPC & International Journal of Engineering \& Technology \\
Website: $w w w . s c i e n c e p u b c o . c o m / i n d e x . p h p / I J E T$ & Research Paper \\
\hline
\end{tabular}

\title{
Development of e-sport platform based on virtual reality with functionality
}

\author{
Yong Ho Kim ${ }^{1}{ }^{*}$, Gul-Won Bang ${ }^{2}$ \\ ${ }^{1}$ Dept. of Self-designed and Open Majors,277Hyodeok-ro, Gwangju University, Nam-guGwangju city, 61743, KOREA \\ ${ }^{2}$ Dept. of Cyber Security \& Police, 277 Hyodeok-ro, Gwangju University, Nam-gu, Gwangju city, 61743, KOREA \\ *Corresponding author_E-mail: 1multi_kyh@gwangju.ac.kr
}

\begin{abstract}
Background/Objectives: National health promotion through physical activities and expanding social participation as well as improvement of public welfare are emerging as important factors in meeting the needs of the times.Government policy and support measures for invigoratingphysical activities of the disabled and the aged are required in public interest. Therefore the development of e-sport machines combined with interest and its contentsis required as not only an infrastructure of physical activity which is able to be national sports as one man one sport but a new sport core technology.

Methods/Statistical analysis: Eco-friendly device is adapted for realizing the sense of power of exercise in consideration of sense of using device like a real play and the effect of exercise. Background and character which reflect sport stadium and participating player to enhance a reality and color is realized. Color design for affective appraisals of the elderly is used.

Findings: This study makes it possible not only to measure cognitive dysfunction such as dementia of the aged and memory deterioration but to develop the game which can be improved cognitive function through the measurement but to contribute national health improvement. It will be also considered to be able to secure baseline data in development environment forvirtual reality game product, including kinesthetic sense against the national game industry concentrated in online game. The development of tangible content and interface technology based on physical and psychologicalcondition ofthe aged also makes it possible to use indicator for requiring content in silver industryas well as development of sporting goods.It will be utilized data for the development of the applied technique of realistic content with functionality.

Improvements/Applications: The game of sports is further developed after considering physical condition of the each elderly andit is expected to develop popular indoor sports and rehabilitation program.
\end{abstract}

Keywords: E-Sport; Functional Game; Haptic; HMD; Realistic Contents; VR; Motion Sensing

\section{Introduction}

Convergence trends of the diffusion technology in sport technol$\operatorname{ogy}(\mathrm{ST})$ is become to make use of IT technology in the various industry fields due to the fusion between a variety of different sports and IT as well as bio technology in the present [1], [2]. In the result, sport technology as successor to BT, NT, and CT has been appeared. Information technology in the sports industry is provided not only judging the events as well as its recording but also systematic and scientific set of training data for athletes, contributing greatly to the improvement of the completion skill. Tangible sport that is able to enjoy existing indoor games which applies technique of IR and VR is on the increase recently [3], [4]. A variety of sport program for the health care system to enjoy the quality life in the elderly only, especially those physical activities have been restricted is greatly demanded. Therefore, the necessity of development for sport technology program that is able to meet the face has been increasing year by year. It is expected that senior-friendly industry, including sport industry will be increased drastically5. Functional game amongst them is a major core field to be able to lead next generation technology as well as contents and is expected the faster growth. It is possible to use not only contents which are needed in the silver industry using an interface technology of game and functional tech- nology for the elderly but also the application of index for the development of sports equipment. It can be also used for material in the development of practical use technology for tangible content with functionality [6], [7].

\section{Development of e-sport platform with func- tionality}

\subsection{Development of motion sensing platform}

Development of technology for interface tracking and speed measurement, synchronization to apply sensor data to program, and firmware which is able to analysis and process extraction data from sensing module are developed in this study [8], [9]. There are included in these not only a tennis racket as well as a circuit diagram of the stick for receiving an egg but also basic design of the stick for an receiving an egg. Development procedure of motion sensing platform is shown in Figure 1. 


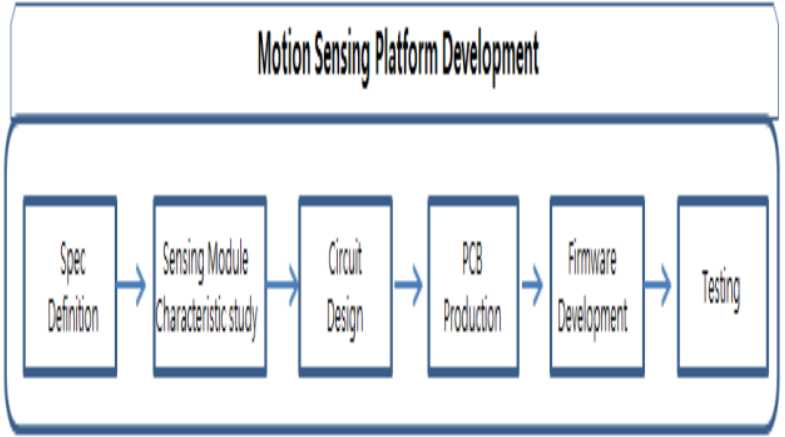

Fig. 1: Development Procedure of Motion Sensing Platform.

\subsection{Making tennis racket}

It is a remote controller used gyro sensor with six-axis to recognize motion of racket for game content. Experiencing impact with the function such asspin direction of ball according to racket motion and calculation of ball rotationas well as haptic function is realistically implemented 10 . Handle segment of racket is created separately.PCB artwork of tennis racket and stick for receiving egg is shown in figure 2 .

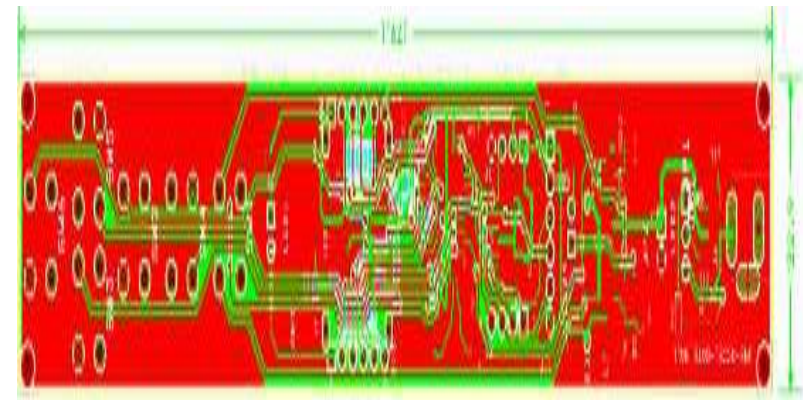

Fig. 2:.PCB Artwork of Tennis Racket and Stick for Receiving Egg.

\subsection{Making stick for receiving egg}

Remote controller for game content can be installed in the center of handle segment for tennis racket. A slope of stick can be adjustable precisely to left and right. The screen of making stick for receiving egg is shown in figure 3 .

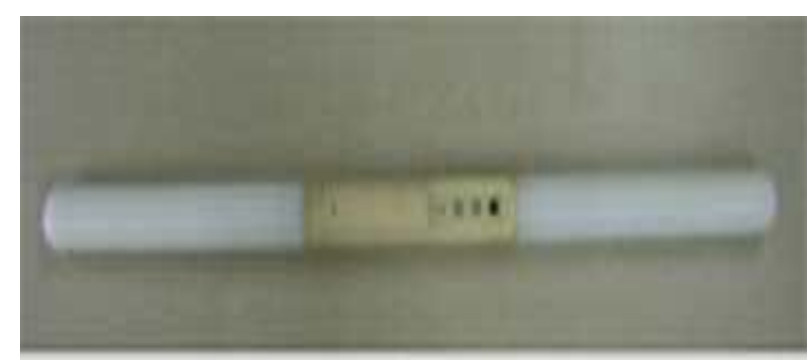

Fig. 3:.Output of Receiving Egg Stick.

\subsection{Making mat for receiving egg}

Data communication through USB and power supply is possible and it is composed of five position indicator, position awareness and two function selection button. Setting of mat size is possible in consideration of user movement. The picture of making racket Tennis is shown in figure 4.

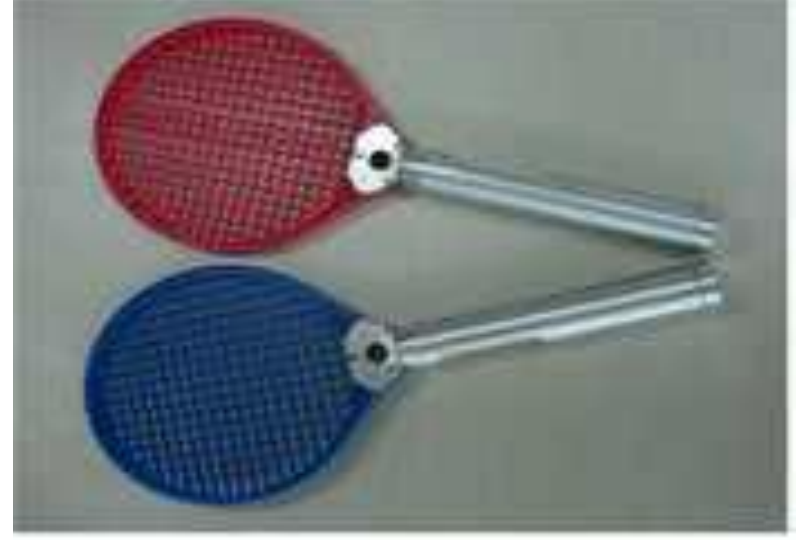

Fig. 4:.Making Tennis Racket

The picture of making game mat is shown in figure 5 and its PCB circuit drawing is shown in figure 6.

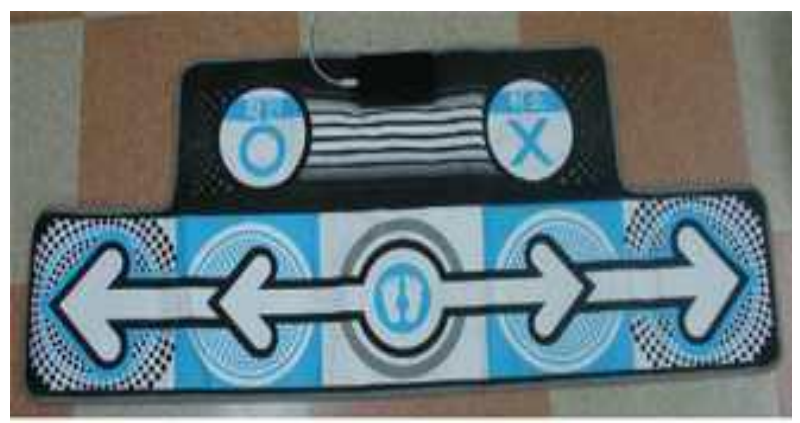

Fig. 5: Picture of Making Game Mat.

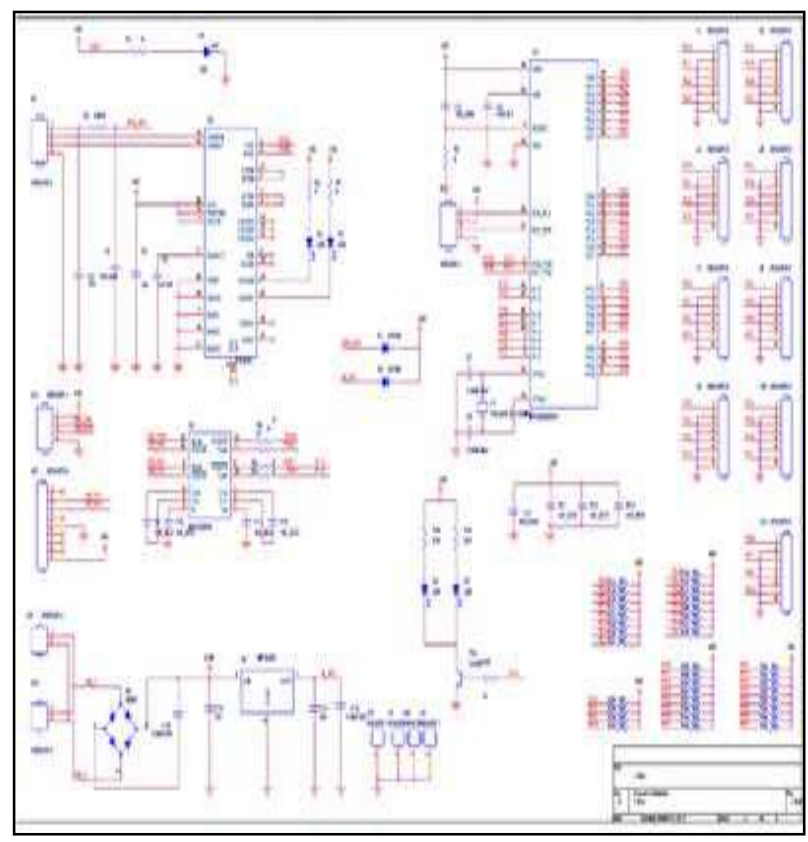

Fig. 6: PCB Circuit Drawing of Game Mat.

Flow chart algorithm of receiving egg game is shown in figure 7. 


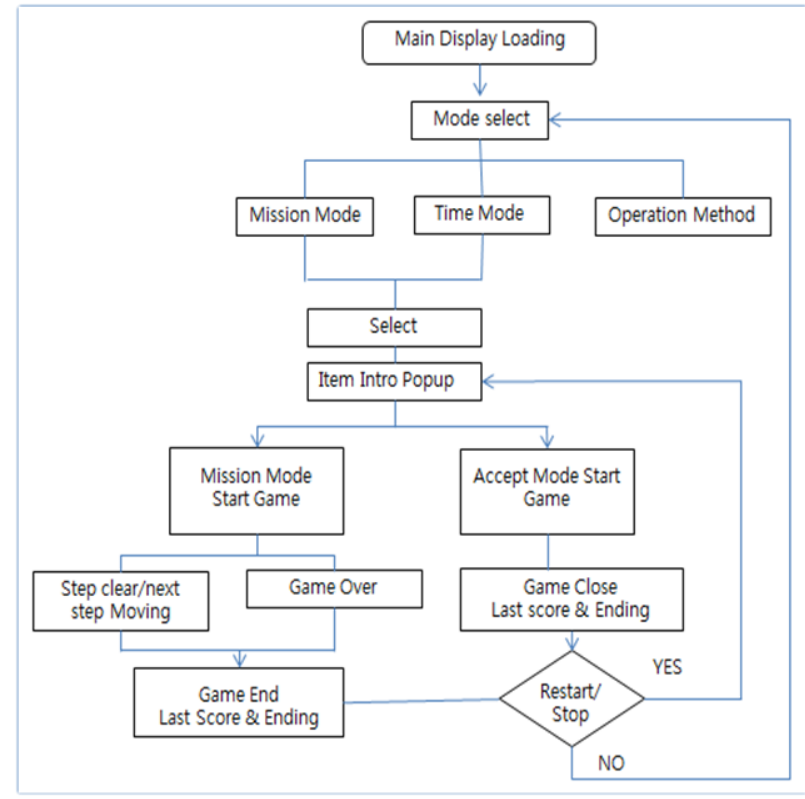

Fig. 7: Algorithm For Receiving Egg Game.

\section{Realization of game algorithm}

Communication method between Interface program and Flash is used basic format in XML. Data in callback function which is already registered in Flash is received and processed in case of transmitting data in the Interface program. The structure of XML is shown in figure 8 .

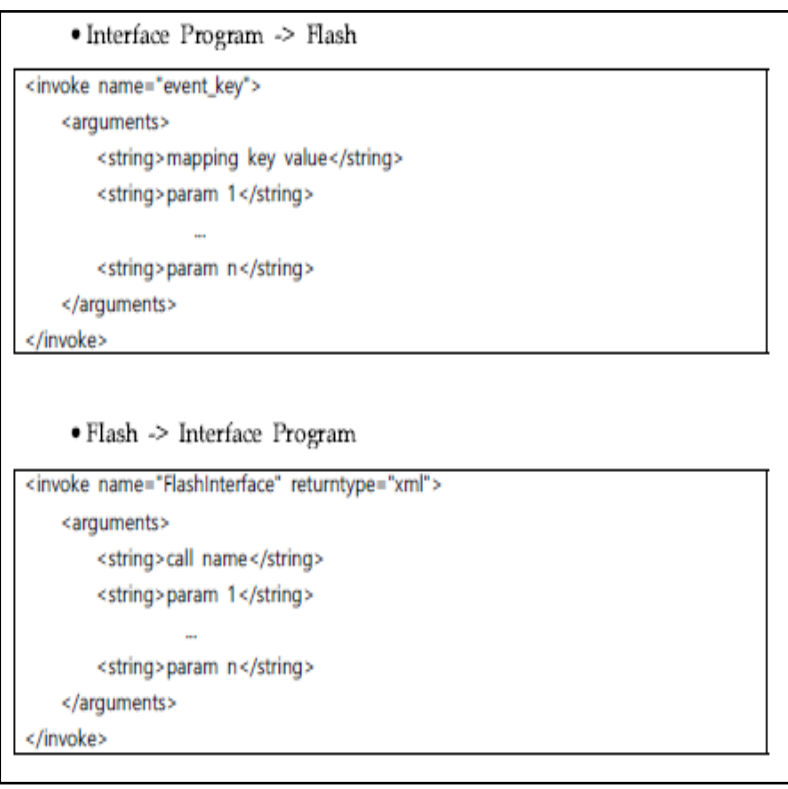

Fig. 8: Xml Structure.

Virtual serial port will be allocated to start communicating if mat in data acquisition and normalization is connected with USB. Data is continuously receiving from mat using predefined protocol. Switch events is generated to deliver Flash if contact switch appropriate to mat is stepped on foot. Flow chart is shown in figure 9.

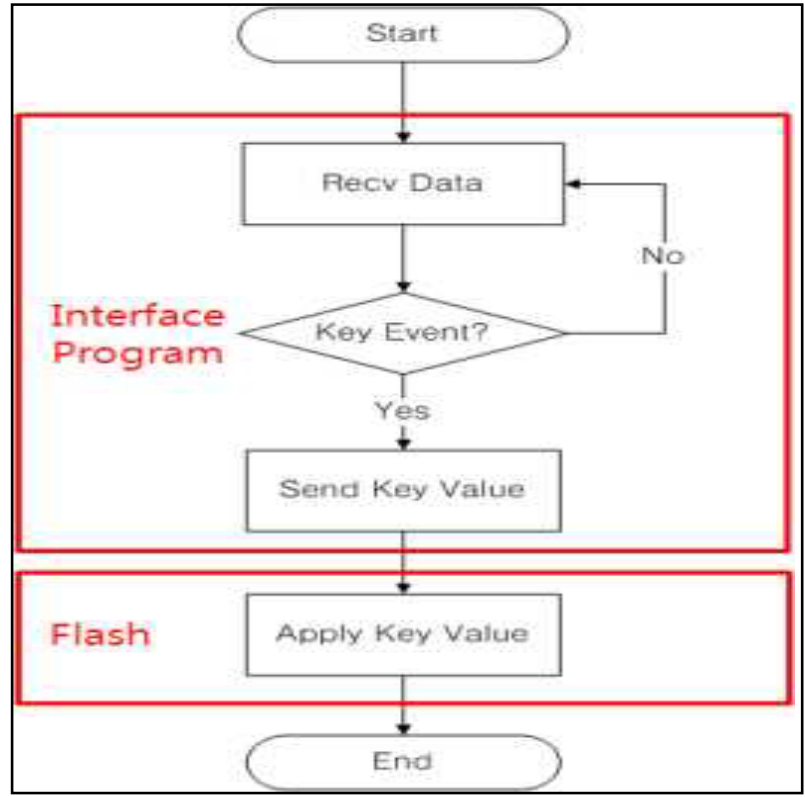

Fig. 9: Flowchart.

Wireless communication between computer and remote controller is used Bluetooth module. Acceleration sensor data in remote controller is processed to convert motion information and Kalman filter is applied not only to normalize acceleration sensor databut to remove noise. Kalman filter is used to find signal amongst noise in order to properly predict how system changes Future condition, as well as current and past, can be can be estimated the optimal value. It is often used as a satellite navigation system, missile trajectory estimation and radar. A highly complicated real time processing system has many practical applications to use due to the most recent developments in microprocessor.

Motion of remote controller is detectable using vector length as a method of motion recognition. Threshold is to be set up to adjust motion sensitivity of remote controller. Motion is considered to be if vector length is larger than Threshold and then motion information is transmitted to Flash. Flow chart of motion recognition is shown in figure 10.

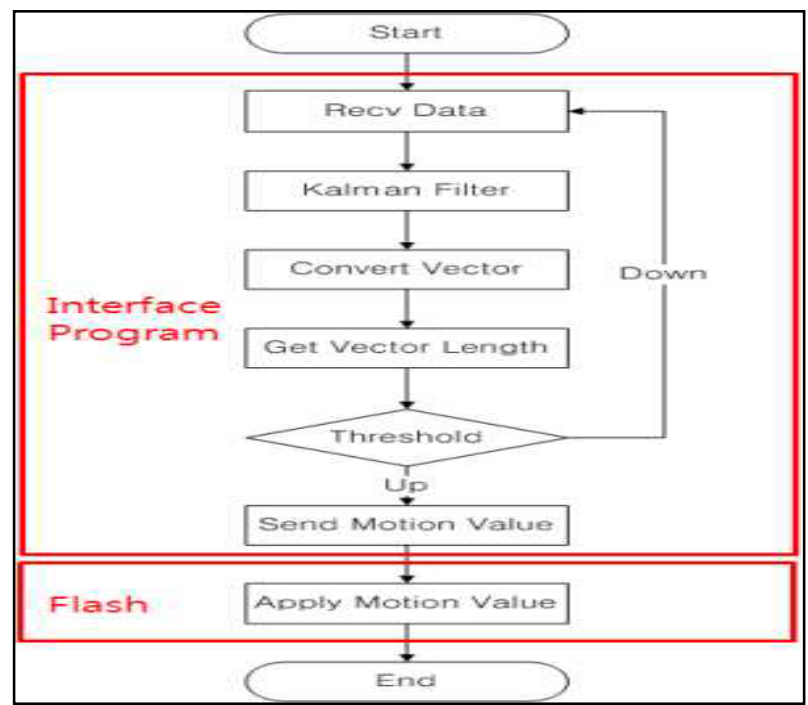

Fig. 10: Flowchart of Motion Recognition.

Demonstration of content through making hardware and interface is shown in figure 11 as a final output. 


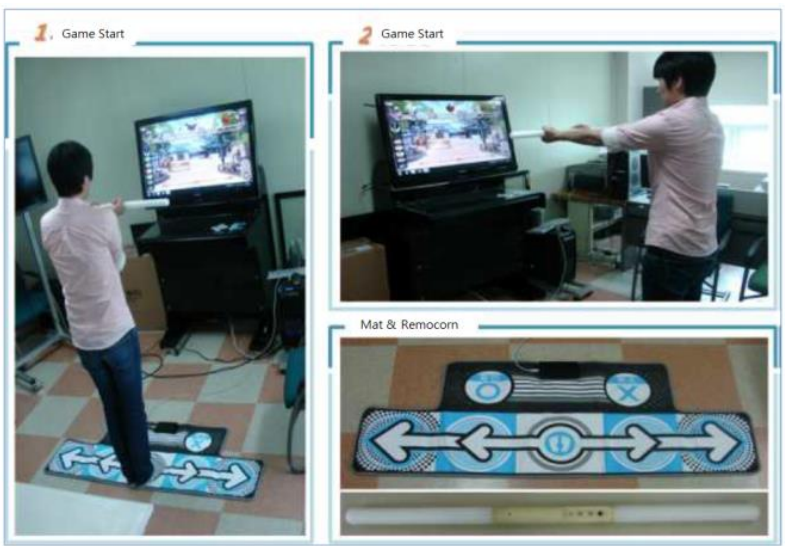

Fig. 11: Content Demonstration through Making Hardware and Interface.

\section{Conclusion}

Remote controller which can recognize motion through the design and development of virtual reality sport equipment with functionality in consideration of characteristic for the disabled and the elderly is implemented in this study. Maintenance of balance and footing plate based on location awareness are developed. Development of VR sport platform which can interact with user interlocking hardware and content are implemented. This study makes it possible not only to measure cognitive dysfunction such as dementia of the aged and memory deterioration but to develop the game which can be improved cognitive function through the measurement but to contribute national health improvement. It will be also considered to be able to secure baseline data in development environment for virtual reality game product, including kinesthetic sense against the national game industry concentrated in online game. The development of tangible content and interface technology based on physical and psychological condition of the aged also makes it possible to use indicator for requiring content in silver industry as well as development of sporting goods. It will be utilized data for the development of the applied technique of tangible content with functionality.

\section{Acknowledgment}

This Study was conducted by research funds from Gwangju University in 2018 , Korea

\section{References}

[1] Shapiro, Stephen L., Dwyer, Brendan, Drayer, Joris, Examining the Role of Price Fairness in Sport Consumer Ticket Purchase Decisions.Sport marketing quarterly, 2016, 25(4),pp.227.

[2] Karande, Anup, Gotmare, V. D. , Shaikh, Javed, Development of High end Textile Structures for Active Sport wears applications using Coating Technology.MAN MADE TEXTILES IN INDIA, 2016,44(9), pp.326-331.

[3] Sassenberg, A., Effects of Sport Celebrity Transgressions: An Exploratory Study.Sport marketing quarterly, 2015, 24(2), pp.78-90.

[4] Ijaz, Kiran, Wang, Yifan, Milne, David, Calvo, Rafael A., VRRides: Interactive VR Games for Health,Lecture Notes in Computer Science. 2016, 2016(9894), pp.289-292.

[5] Kim, Sangil, Technology Roadmap for Realistic Contents: The Korea Case,COMMUNICATIONS IN COMPUTER AND INFORMATION SCIENCE. 2016, 2016(617), pp.58-63.

[6] Ji, Y. , Ruobing, Q. , Bin, L., Functional connectivity of temporal parietal junction in online game addicts: a resting-state functional magnetic resonance imaging study,(National medical journal of China,Zhonghua Yixue Zazhi ). 2014,94(4),pp.372-375

[7] [7] Luth, C., Haskell in Space: An interactive game as a functional programming exercise,JOURNAL OF FUNCTIONAL PROGRAMMING. 2003, 13(6), pp. 1077-1086.

[8] Shaw, M. L., Motion Sensing Techniques and Analysis for Direct Tire Pressure Monitoring,SAE Special Publications(SAE SP). 2003 $-(1782)$, pp.35-44.
[9] Lin, C.-Y., Lin, M.-C. , Chen, S.-H., Motion Sensing Technology on Rehabilitation for Children with Physical Disabilities,Lecture Notes in Computer Science. 2013, -*8011), pp.500-507.

[10] Salisbury, C. , Gillespie, R. B., Tan, H. Z. , Barbagli, F. , Salisbury, J. K., What You Can't Feel Won't Hurt You: Evaluating Haptic Hardware Using a Haptic Contrast Sensitivity Function, IEEE TRANSACTIONS ON HAPTICS. 2011, 4(2), pp.134-1412.Thakurdesai PA, Kole PL \& Pareek RP (2004), Evaluation of the quality and contents of diabetes mellitus patient education on Internet. Patient Education and Counseling 53, 309-313. 